\title{
Are All Prominences Either Sinistral or Dextral?
}

\author{
J.B. Zirker
}

National Solar Observatory, Sunspot, N.M. 88349, USA

E-mail: zirker@noao.edu

\section{J.-L. Leroy}

\section{I.A.C. - T.H.E.M.I.S, E-38200 La Laguna, Tenerife, Canary Islands, SpainE-mail:leroy@themis.iac.es}

\section{Gaizauskas}

Herzberg Institute of Astrophysics, National Research Council of Canada, Ottawa K1A OR6, Canada

\begin{abstract}
A reanalysis of the prominence magnetic field measurements obtained by Leroy et al. (1983) indicates that only a small fraction of these prominences did not obey the chirality rules they discovered and that Martin et al. (1994) confirmed.
\end{abstract}

\section{Introduction}

Martin, Tracadas and Billamoria (1994, hereafter MTB) rediscovered a regularity first uncovered by Leroy, Bommier and Sahal-Brechot (1983). Sinistral (dextral) filaments with axial fields pointing to the left (right) as viewed from the neighboring positive magnetic field region, predominate in the southern (northern) hemisphere. This preference is independent of the solar cycle number.

MTB's hemispheric rule is only statistical; of 72 filaments, $11 \%$ were exceptions to the rule. Active region filaments do not show such a regularity. Filaments bordering active regions show only a slight tendency.

MTB also found that the orientation of the "barbs" or "legs" of quiescent filaments is a reliable indicator of the sinistral/dextral characteristic. Dextral filaments were invariably "right-bearing" and sinistrals invariably "left-bearing" whenever both characteristics could be determined with confidence.

MTB inferred the direction of the axial fields from the appearance of the $\mathrm{H}_{\alpha}$ plagettes near the filaments. Although this method yields consistent results, direct measurements of the axial field components would be more objective. Fortunately, we can use the data set of Leroy et al. (1983, 1984, hereafter LBS) to make an independent check on the results of MTB.

LBS measured the depolarization of the HeI D3 line (the Hanle effect) in 256 prominences at the solar limbs, during summer campaigns from 1974 to 1982. Their analysis yields two possible field vectors, symmetric about the line of sight. In many but not all cases, the two vectors have opposite components along the axis of the prominence, a $180^{\circ}$ uncertainty in the axial field direction. 
In this paper we reanalyzed the data set of LBS, selecting favorable cases in which both possible field vectors have the same direction of the axial component. In such cases an unambiguous classification of a filament as sinistral or dextral is feasible. Full details of our procedure can be found in Zirker et al. (1997).

We also report on an investigation of barb orientation as an indicator of the sinistral/dextral character.

\section{Results for the LBS Data Set}

Of 256 prominences in the 1984 catalog, 50 were in active regions and were excluded from this study. Of the remaining 206,131 permit a definite classification. Of these 131 prominences, 36 do not appear on synoptic $\mathrm{H}_{\alpha}$ maps, so that their orientations to the line of sight (a necessary piece of information) could not be determined.

Of the remaining 95 prominences, only four disobey the hemispheric rule. We examined the four exceptional cases to find a common factor, without success.

\section{A Test of the Barb Regularity}

We searched the film archives of the Ottawa River Solar Observatory (ORSO) for the 95 classifiable filaments.

In the years $1978,1979,1980$ and 1982,53 filaments were classifiable in the LBS data set. Of these, 32 appeared in the ORSO data set. Of these 32 only 12 were classifiable as sinistral or dextral from the arrangement of their barbs. Many filaments had barbs extending normal to the filament axis, and others had only one or two barbs with definite inclinations. Polar crown filaments usually lacked sufficient fine structure to be classified. Of the 12 classifiable filaments, we found that three disobeyed the hemispheric rule.

\section{Conclusions}

This study confirms and strengthens the results of MTB. Quiescent filaments and filaments at the borders of active regions obey the hemispheric rule with only a few exceptions. However, exceptions do exist and, so far, we have no explanation for them.

Our attempts to test barbs as an indicator of sinistral or dextral character were hampered by too small a sample. We recall, however, that MTB found a one-to-one correlation whenever a reliable comparison was possible.

\section{References}

Leroy, J-L., Bommier, V. and Sahal-Brechot, S. 1983, Sol. Phys., 83, 135

Leroy, J-L., Bommier, V. and Sahal-Brechot, S. 1984, A\&A, 131, 33 
Martin, S.F., Billamoria, R. and Tracadas, P.W. 1994, in Solar Surface Magnetism, (ed.) R. Rutten and C.J. Schrijver, Kluwer Acad. Publ., Dordrecht, Holland, p. 339

Zirker, J.B., Martin, S.F., Harvey, K. and Gaizauskas, V. 1997, Sol. Phys., 175, 27 